\title{
Neither Incomplete nor Non-Local, Just Entangled
}

\author{
Franz Kohl \\ Independent Researcher, Vienna, Austria \\ Email:kohl@data.isas.tuwien.ac.at
}

How to cite this paper: Kohl, F. (2021) Neither Incomplete nor Non-Local, Just Entangled. Journal of Quantum Information Science, 11, 143-155.

https://doi.org/10.4236/jqis.2021.114012

Received: October 6, 2021

Accepted: December 6, 2021

Published: December 9, 2021

Copyright (c) 2021 by author(s) and Scientific Research Publishing Inc. This work is licensed under the Creative Commons Attribution International License (CC BY 4.0).

http://creativecommons.org/licenses/by/4.0/

\begin{abstract}
Bell-state experiments with pairs of polarization-entangled photons are interpreted without any hinge on non-local mechanisms. The presented model rests on a careful analysis of published experimental findings. These foundations are implemented into a standard quantum mechanical treatment that obeys the purely local nature of each polarization preparation in the course of a measurement process. Polarization entanglement is ascribed to the generation of indiscernible photon pairs while undistorted propagation maintains this interrelation. Thus, the proposed approach assigns the essential characteristics of polarization entanglement to each constituent of an entangled pair. Accordingly, space-time separated polarization preparations lead to consistent probabilities of joint detection events. The obtained results agree with those of previous non-local models and thus reproduce the experimentally required violations of the Bell inequality. Since the presented approach lacks any non-local phenomenon, hidden variables are rendered superfluous, too.
\end{abstract}

\section{Keywords}

Quantum Non-Locality, Polarization Entanglement, Bell Experiments

\section{Introduction}

In 1935, Einstein, Podolski and Rosen (EPR) questioned the completeness of quantum theory regarding the description of physical reality [1]. Their reasoning involves constituents of entangled states that have sufficiently spread in space before completely disjointed measurements of non-commuting properties will take place. In response to EPR, J.S. Bell provided in 1964 a general criterion for the results of disjoint measurements. If space-time separated measurements imply statistical independence of related measurement results, basic findings concerning entangled-state experiments cannot be met even with the aid of hidden va- 
riables [2]. Bell's approach was expanded considerably by Leggett leading to comparable results [3].

In contrast, the so-called non-local quantum-mechanical explanation of the experimental facts claims successful modeling of associated phenomena [4]. However, this approach implicitly equates local state preparations with ordinary measurements imposed on the complete entangled state, despite any spacetime spread. Accordingly, state preparations applied locally to one particle of an entangled system provoke the instantaneous collapse of the system's wavefunction in entire space [5]. This non-local feature of quantum mechanics encounters no aftermath at all. The non-local explanation of Bell-type experiments, however, involves the instant creation of a new wave-function describing the properties of the remaining particle in entire space. In particular, the new wavefunction must be in line with the parameters of the preceding, space-time-separated state preparation. Hence because of previous entanglement, the first state preparation bears physical consequences that are not subjected to special relativity.

In spite of this peculiar feature, the related quantum-mechanical formalism leads to a persuasive agreement with all experimental observations on the investigated property, e.g. the photon polarizations. Experimental evidence was presented for diverse particles like photons or atoms and distinct characteristics like spin or polarization. Therefore, a vast number of scientific papers have adopted the non-local view. Furthermore, it is broadly accepted that the concept of local reality as attributed to EPR can be refuted by Bell's criterion. Nevertheless, the lack of any physically satisfactory model of such a superluminal link between space-time separated measurements causes revenant debates about the non-locality construct [5] [6] [7]. In fact Erwin Schrödinger addressed his repugnance regarding this non-local ingredient of the rejection of EPR arguments [8].

Recently, R. Griffiths showed in an in-depth quantum theoretical discussion of the topic that the non-locality claim is incompatible with Hilbert-space quantum mechanics [9]. Moreover, a Bell-type experiment employing circularly polarized photons was explained recently without any apparent reference to nonlocal phenomena [10].

Statistical independence of measurement results in case of space time separated measurements is a further essential of the Bell criterion [11]. However, while the local measurement conditions can be chosen in a statistical independent manner, the entangled objects measured may carry a statistic interrelation themselves. In that case, the observed extraordinary correlations can be explained without the use of further ingredients like hidden variables.

In this work, the essential characteristics of polarization-entangled, space-time separated photons are deduced from present experimental evidence. Thereby, a local framework of joint polarization preparations of entangled photons could be derived in agreement with the experiment. Hence, non-local explanations of related observations are dispensable. 


\section{Polarization Entangled Photon Pairs}

A type-I SPDC Bell state source delivers polarization-entangled photon pairs according to

$$
\Psi_{I}=\frac{1}{\sqrt{2}}\left(|H\rangle_{1}|H\rangle_{2}+\mathrm{e}^{i \theta}|V\rangle_{1}|V\rangle_{2}\right)
$$

that propagate in different directions. Here $|V\rangle,|H\rangle$ denote orthogonal polarization states, the subscripts indicate indiscernible photon instances and $\theta$ denotes an experimentally adjustable phase shift that is preferably chosen $\theta=0$ or $\theta=\pi$. In contrast, type-II SPDC yields

$$
\Psi_{I I}=\frac{1}{\sqrt{2}}\left(|V\rangle_{1}|H\rangle_{2}+\mathrm{e}^{i \theta}|H\rangle_{1}|V\rangle_{2}\right)
$$

as used in reference [12]. Typical Bell state experiments manage to guide the two photons in opposite directions to maximize their space-time separation. In case of free space photon propagation, the polarization states $\Psi_{I}, \Psi_{I I}$ remain valid while their spatial extension expands with up to $2 c_{0} \cdot t$ in space. Hence after space-time separation of the photons, ordinary measurements on the entire systems (1) or (2) seem to be impossible. Therefore, it is common practice to assemble a posteriori the results of polarization state preparations imposed separately on space-time separated, single photons. These compilations are exploited as if they were the result of ordinary quantum-mechanical measurements on the entangled systems $\Psi_{I}, \Psi_{I I}$ in spite of the fact that each separate polarization preparation distorts these states [13]. Therefore, particular skepticism is mandatory if the rules of quantum-mechanical measurements are employed in conjunction with partial investigations on spatially spread systems.

Equations (1) and (2) specify only the polarization subspace of the entangled systems, a restriction that renders valid predictions regarding the polarization manipulations during Bell-experiments. This success tempts to derive a complete picture of the evolution of the systems $\Psi_{I}, \Psi_{I I}$ based on a restricted foundation. The incomplete use of the Hilbert space of the entangled two-photon system seemingly induces the need for a non-local wave-function switch, i.e., the required collapse of the wave-function of the entangled system must be accompanied by an instant birth of a single-photon state in entire space. As all non-local explanations of the experiments lack of undisputed physical grounds, the interpretation of Belltype experiments ask for a rigorous reconsideration, as given below.

\section{A Thorough Local Explanation of Experimental Results}

The following treatment circumvents the state specifications (1) or (2). They certainly apply to the emission of polarization-entangled, indiscernible photons. In case of different propagation directions, however, polarization-entangled photons are clearly distinguishable even if their polarization entanglement and thus the representation by (1) or (2 remains unaffected. As polarization preparations arise through exclusive interaction of each constituent of (1) or (2) with its asso- 
ciated polarizer, Bell experiments also inhere particle discrimination. Therefore, the following approach rests on the view of two separate, but polarization entangled quantum objects.

In the following discussion, the subscripts $\mathrm{L}$ or $\mathrm{R}$ refer to Figure 1 and denote the target locations left or right of the source of entangled photons, respectively. The characters $H$ and $V$ designate "vertical" and "horizontal" polarizer channels as well as their related detectors. The quotes indicate that the ordinary and extraordinary rays of the polarizers apply to supposedly rotated photon polarizations occurring at the outputs of both polarization modulators. Since $H$ and $V$ are prepared after an arbitrary polarization rotation by the adjacent polarization modulators, they do not directly correspond with the notion of the entangled states (1) or (2). Furthermore, bold characters $\boldsymbol{V}, \boldsymbol{H}$, and $\boldsymbol{\varphi}$ specify 2D directions and the orientation of photon electric field amplitudes, respectively. Bold face is omitted if scalar angles are meant.

\subsection{Key Experiments}

Starting point of the following line of arguments are common findings of numerous experiments on photon polarization entanglement. In fact, a prime example [4] [12] that is sketched in Figure 1 is exploited for this purpose. The related measurement setup characterizes polarization manipulations on entangled photons after their space-time separation. In contrast to numerous others, this investigation is described in an exemplary manner and all experimental results necessary for re-evaluation were publicly accessible from the Author of Ref. [12]. The complete measurement setup of Figure 1 generates two preparation results at corresponding moments in time but there is no instant cognition of both space-time separated detection events possible anywhere in space. The practical solution is to record the detection events aside with precise timestamps separately at sites $L$ and $R$. After finishing a complete measurement run, joint events are compiled from the recorded data for further analyzes.

\subsection{The Experimental Foundations}

All known experiments with polarization-entangled photons proof the violation

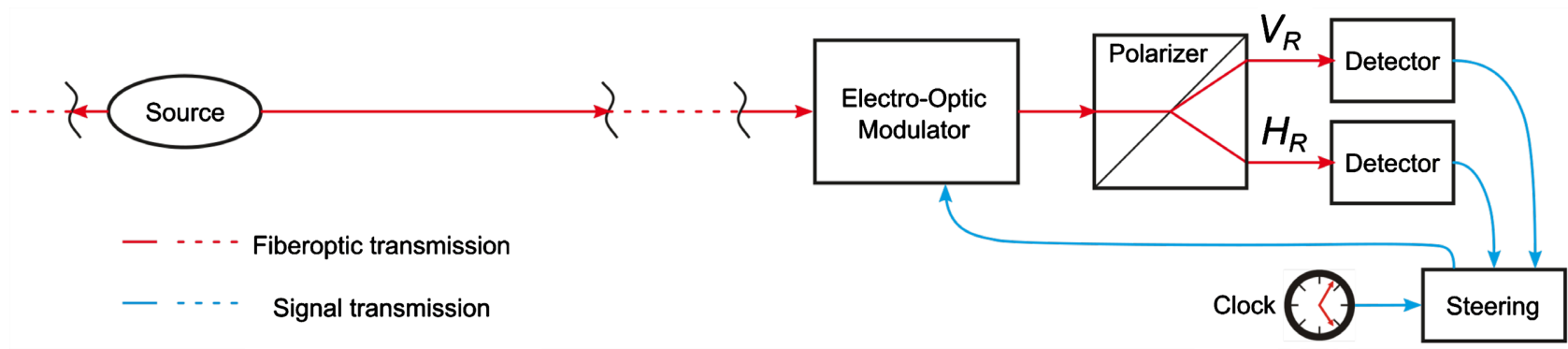

Figure 1. Schematic view of the right half of the symmetric setup used in the experiment by G. Weihs et al. [4] [12]. The electro-optic modulator rotates the polarization of the photons traveling rightwards according to a random setting by the right steering unit. The modulator-polarizer combination acts as a rotatable polarizer which prepares the photon polarization into a rotated vertical $\left(V_{R}\right)$ or horizontal $\left(H_{R}\right)$ one. 
of Bell's inequality. Apart from practical limitations, the published results are in accordance with the findings listed below where type-I SPDC, i.e. parallel polarization of the entangled photons, is presumed.

1) The chance to obtain a $V_{L}\left(V_{R}\right)$ preparation with a polarization analyzer equals the probability to obtain a $H_{L}\left(H_{R}\right)$ result, i.e.

$p\left(V_{L}\right) \approx p\left(H_{L}\right) \approx p\left(V_{R}\right) \approx p\left(H_{R}\right) \approx 0.5$, no matter the rotation of the polarizers around the propagation direction is. The orientation of the left and right polarization analyzer $\alpha_{L}$ and $\beta_{R}$, respectively, are referenced to a common cartesian frame.

2) In case of perfectly aligned, ideal polarization analyzers, joint $V_{L}, V_{R}$ or $H_{L}$, $H_{R}$ detection events are perfectly correlated. Hence, combinations like $V_{L}, H_{R}$ are not observed. The term joint detections refer to pairs of entangled photons exclusively. The pairwise determinism must be attributed to the emission of indiscernible, polarization-entangled photons. Indiscernibility predetermines the polarization domain of the Hilbert space for each generated pair of entangled photons. It also forces equal response to equivalent polarization preparations. Undistorted propagation in space make entangled photons discernible at least because of their spatial separation. However, separation in free space does not affect the polarization domain, maintaining the characteristica of polarization entanglement over arbitrary distances.

3) In case of different orientations of the polarizers, $\alpha \neq \beta$, the probabilities of joint detections are determined exclusively by the angular difference of the polarizer alignments. Moreover, only statistical probabilities of the four pairwise detection events can be given.

4) Even for aligned polarizers, the outcomes of subsequent joint detections of a series of entangled pairs show no correlation.

5) All joint probabilities valid for orthogonal polarization entanglement (typeII SPDC) can be obtained from their SPDC-I counterpart by simply replacing a $V(H)$ preparation by an $H(V)$ on either the $L$ or the $R$ side of the experiment.

From the experimental facts listed above, the unique pairwise property of polarization entangled photons finding 2) is identified as their essential characteristic. A comparison with ordinary polarized light illustrates the extraordinary consequences of entanglement. In case of pairwise picked photons extracted from a stream of perfectly linear polarized light, corresponding preparation results are the absolute exception. Such a correlation occurs only if photon polarization and both analyzer alignments coincide but then with certainty, i.e. alternative outcomes will never occur. Any deviation from mutual alignment decomposes the joint results of separate polarisation preparations into four stochastic quantities.

With aligned perfect analyzers, streams of entangled photons yield exactly two alternatives of pairwise preparation outcomes. Therefore, the observations on polarization entangled photon pairs are incompatible with any prescribed linear polarization. In that case, ordinary photon pairs yield four combinations of pair- 
wise joint preparation results instead of two. The extraordinary behavior of the constituents of each polarization entangled pair must be attributed to their indiscernible polarization property. Again, for deviating analyzer alignments the joint preparation results end up in four stochastic quantities. However the ruling probabilities will differ from the case of linear polarized light.

\subsection{The Local Model}

The local explanation assumes that both entangled photons to be measured have unknown but identical (orthogonal) polarization prior to the state preparations by the space-time separated polarization analyzers. A virtual variant of the above experiment may help to illustrate remarkable features of the spatial spreading of the entangled photons of states (1) and (2). Assume that only one photon of each entangled pair is analyzed, e.g., at $\mathrm{R}$, in accordance with the setup sketched above while the other one serves as a herald only and arrives its dedicated detector without intermediate polarization manipulation. Of course, different propagation delays have to be known in advance to enable heralding. An appropriate time window can be used to discriminate all detections of entangled photons beyond the polarization analyzer from unspecific detection events.

In accordance with finding 1), equal probabilities $p\left(V_{R}\right)=p\left(H_{R}\right)=0.5$ should result independently of the chosen orientation of the polarization analyzer at $R$. Because of symmetry, $p\left(V_{L}\right)=p\left(H_{L}\right)=0.5$ also would hold if the functions of the $L$ and $R$ sides are interchanged.

Accordingly, three important consequences follow:

First, equal partitioning of $V_{R}$ and $H_{R}$ detections for any analyzer orientation implies that the respective photons leave the source with unpredictable polarization $\boldsymbol{\varphi}$. Moreover, the stream (ensemble) of entangled photons lacks any distinguished polarization angle $\varphi$. Second, equal partitioning is also independent of the sequence of detection instances of the entangled photons. Therefore, the heralding photons may be detected next to the source or registered much later after traveling a distance toward its detector that may exceed the traveling distance of the analyzed photons. Third, since a previous registration of the herald will not affect the observation at $R$, it doesn't matter whether or not the entangled states (1) or (2) still exist right at the moment of the $R$ measurement.

Reverting to the complete setup suggested by Figure 1, related experiments unveiled that in case of aligned polarization analyzers, both entangled photons undergo with certainty only selected combinations of polarization preparations apart from possible exceptions due to experimental imperfections. This observation must be attributed to strict intra-pair polarization relations of indiscernible photons, i.e., considered as a unique feature of entanglement. Such certainty is not to be expected from a split stream of linear polarized photons when the photon polarization deviates from the setting of the aligned analyzers. In case of type-I SPDC the registrated combinations are $V_{L} \wedge V_{R}$ or $H_{L} \wedge H_{R}$ while for type-II SPDC the combined detections $V_{L} \wedge H_{R}$ or $H_{L} \wedge V_{R}$ are predetermined, 
where $\wedge$ denotes the logical and.

The entanglement-induced intra-pair determinism contrasts with the interpair randomness dictated by the unpredictable sequence of polarization angles $\boldsymbol{\varphi}$. The latter follows from the definitions of states $\Psi_{I}$ or $\Psi_{I I}$ which forbid polarization restrictions regarding the stream of SPDC photons. Hence the local sequences of detection results at $L$ or $R$ are random even for aligned polarizers.

To summarize, polarization entanglement comprises pairwise linked, but sequentially indeterminate polarization states $\left|\varphi_{n}\right\rangle_{L},\left|\varphi_{n}\right\rangle_{R}$ of the photons propagating toward $L$ or $R$ with

$$
\left|\varphi_{n}\right\rangle_{L} \|\left|\varphi_{n}\right\rangle_{R} \text { or }\left|\varphi_{n}\right\rangle_{L} \perp\left|\varphi_{n}\right\rangle_{R}
$$

for photons produced by type-I SPDC or SPDC-II, respectively. The subscript $\mathrm{n}$ is used here to highlight the affiliation to a single instance of $\Psi_{I}$ or $\Psi_{I I}$. Furthermore, shorthand notation is applied, e.g., $\left|\varphi_{n}\right\rangle_{L}$ represents the polarization state of the $n^{\text {th }}$ left-propagating photon $\left|\boldsymbol{\varphi}_{n}\right\rangle_{L}=\cos \varphi_{n, L}|\boldsymbol{x}\rangle+\sin \varphi_{n, L}|\boldsymbol{y}\rangle$, where $\boldsymbol{x}, \boldsymbol{y}$ form an orthonormal basis in the polarization subspace and the inclination $\varphi_{n, L}$ is referred to the $\boldsymbol{x}$-axis.

There is no direct correspondence of the states of (3) with the photons of the entangled states (1) or (2): $\Psi_{I}$ or $\Psi_{I I}$ treat the emergence of each pair of polarization-entangled photons as a single physical aggregate. In contrast, relations (3) deal with individual photons propagating either toward $L$ or $R$ as signaled by the subscripts. However, one could infer that polarization entanglement ensures fixed polarization interrelations (3).

The polarizers and detectors of the experiment of Figure 1 are kept fixed in space. Nevertheless, according to $[4,12]$, each sequence of polarization modulator and beam-splitting analyzer can be conceived as a compact rotary polarization analyzer. Figure 2 depicts a tentative example of polarization analyzer alignments aside with an exemplary polarization angle $\varphi_{n}$ of the SPDC-I entangled photon pair. With respect to a common reference direction, we arbitrarily declare effective angles of tilt at the entrances of the modulators, i.e. $\alpha_{L}$ for $V_{L}$ and $\beta_{R}$ for $\boldsymbol{V}_{R}$ of the $L$ and $R$ polarization analyzer, respectively.

Impinging at the entrance face of a polarizer, an electromagnetic wave that is linearly polarized along $\varphi_{n}$ is split in an ordinary and an extraordinary partial wave. The magnitudes of the partial waves depend sinusoidally on the inclinations $\alpha_{L}-\varphi_{n}, \beta_{R}-\varphi_{n}$ of the polarizer's reference directions with respect to the wave polarization $\boldsymbol{\varphi}_{n}$. The polarization directions $\boldsymbol{V}$ and $\boldsymbol{H}$ align with the electric field vectors of the respective partial waves corresponding to the detectors $V$ and $H$ at both the $L$ and the $R$ site of the experiment. Rotations by $\pi$ around the optical axis remain the function of a polarizer unchanged while optical waves acquire opposite phase. Thus, we can arbitrarily limit the considerations to the range $0 \leq \alpha_{L}, \beta_{R}, \varphi_{n}<\pi$.

If not otherwise stated, type-I SPDC, i.e., parallel polarization of each photon pair $\varphi_{n, L}=\varphi_{n, R}=\varphi_{n}$ is implied in the following reasoning. At the level of single 


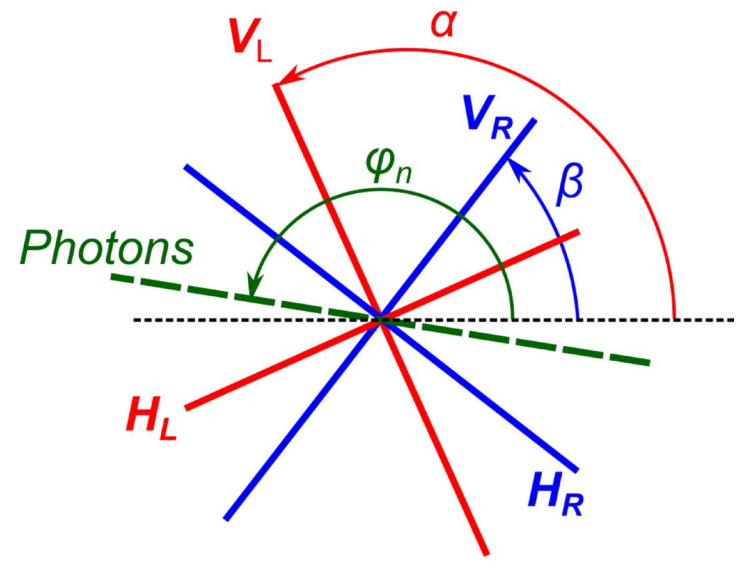

Figure 2. Exemplary polarization of SPDC-I photons at the entrance faces of the polarization modulators. The equivalent orientation of ordinary and extraordinary electromagnetic waves in the polarizers is represented by orthogonal $V$ and $H$ directions while the polarizer sites $L$ and $R$ are indicated by subscripts.

photons, the orientation of their electric field vector affects the probabilities of taking the ordinary or extraordinary path of the polarization analyzer. According to Figure 2, the probability amplitudes of photons of polarization $\varphi_{n}$ to be prepared by the $L$ polarizer in the basis $\left|\boldsymbol{V}_{\alpha_{L}}\right\rangle$ and $\left|\boldsymbol{H}_{\alpha_{L}}\right\rangle$ are given by

$$
\left\langle\boldsymbol{\alpha}_{L} \mid \boldsymbol{\varphi}_{n}\right\rangle=\cos \left(\alpha_{L}-\varphi_{n}\right) \text { and }\left\langle\boldsymbol{\alpha}_{L} \pm \pi / 2 \mid \boldsymbol{\varphi}_{n}\right\rangle=\mp \sin \left(\alpha_{L}-\varphi_{n}\right),
$$

respectively. Accordingly, for the $R$ analyzer channels $V_{\beta_{R}}$ and $H_{\beta_{R}}$ the dot products

$$
\left\langle\boldsymbol{\beta}_{R} \mid \boldsymbol{\varphi}_{n}\right\rangle=\cos \left(\beta_{R}-\varphi_{n}\right) \text { and }\left\langle\boldsymbol{\beta}_{R} \pm \pi / 2 \mid \boldsymbol{\varphi}_{n}\right\rangle=\mp \sin \left(\beta_{R}-\varphi_{n}\right)
$$

apply. Since the local model excludes mutual interference of separated polarization preparations, no phase factor is required. Purely local preparation of photon polarizations implies strict statistical independence of space-time separated preparation events. Consequently, the local treatment of joint detection events should involve the product of the individual probability amplitudes (4), (5).

In case of type-I SPDC and the combined outcome $V_{\alpha_{L}} \wedge V_{\beta_{R}}$, the product

$$
c \cdot\left\langle\boldsymbol{\alpha}_{L} \mid \boldsymbol{\varphi}_{n}\right\rangle \cdot\left\langle\boldsymbol{\beta}_{R} \mid \boldsymbol{\varphi}_{n}\right\rangle=c \cdot \cos \left(\alpha_{L}-\varphi_{n}\right) \cdot \cos \left(\beta_{R}-\varphi_{n}\right)
$$

results for the related probability amplitude for a single entangled pair of photons with common but unknown polarization $\varphi_{n}$. The provisional factor $c$ provides the opportunity of renormalization.

Products of probability amplitudes are common in the treatment of joint quantum-mechanical processes [14] [15]. The double appearance of $\boldsymbol{\varphi}_{n}$ in (6), however, is an intrinsic property of indiscernible, polarization entangled photons. The probability amplitudes in (6) concern corresponding moments in time but separate locations.

Analogue to (6), the joined detection events $V_{\alpha_{L}} \wedge V_{\beta_{R}}$ entail the product

$$
c \cdot\left\langle\boldsymbol{\alpha}_{L} \mid \boldsymbol{\varphi}_{n}\right\rangle \cdot\left\langle\boldsymbol{\beta}_{R} \pm \pi / 2 \mid \boldsymbol{\varphi}_{n}\right\rangle=\mp c \cdot \cos \left(\alpha_{L}-\varphi_{n}\right) \cdot \sin \left(\beta_{R}-\varphi_{n}\right)
$$

in case of type-I SPDC. Including joint detections that comprise $H_{\alpha_{L}}$, there are 
in total four combinations of such joint detection events.

The sketched treatment constitutes a substantial difference to the rejection of the local realistic view by Bell [2], which rests on products of expectation values. That approach is only justified for statistically independent pairwise detection events which is considered by Bell an inherent characteristic of local realism. The lack of mutual interference between measurement processes, however, is not tantamount to statistically independent measurement results. In contrast, (6) and (7) simultaneously entail the statistical independence of local preparation decisions as well as an inherent correlation capability due to the joint photon polarizations $\boldsymbol{\varphi}_{n}$.

To pave the way to joint detection probabilities, all contributing probability amplitudes have to be considered [14]. Accordingly, averaging over all possible photon polarizations $\boldsymbol{\varphi}_{n}$ constitutes the essential step of the local explanation of related experiments. In case of type-I SPDC and $V_{\alpha_{L}} \wedge V_{\beta_{R}}$ joint detections, the integral

$$
\frac{c}{\pi} \int_{0}^{\pi}\left\langle\alpha_{L} \mid \varphi\right\rangle \cdot\left\langle\beta_{R} \mid \varphi\right\rangle \mathrm{d} \varphi
$$

provides this task. Using basic trigonometric identities it is easy to show that (6) and (8) lead to the result

$$
\frac{c}{\pi} \int_{0}^{\pi} \cos \left(\alpha_{L}-\varphi\right) \cdot \cos \left(\beta_{R}-\varphi\right) \mathrm{d} \varphi=\frac{c \cdot \cos \left(\alpha_{L}-\beta_{R}\right)}{2}
$$

Contrary to the assumption of Bell [16], Equation (9) proves setting dependent correlations between local measurement results just because of the entanglement of involved photon pairs. With (7) the average probability probability amplitude for $V_{\alpha_{L}} \wedge V_{\beta_{R}}$ follows from

$$
\frac{ \pm c}{\pi} \int_{0}^{\pi} \cos \left(\alpha_{L}-\varphi\right) \cdot \sin \left(\beta_{R}-\varphi\right) \mathrm{d} \varphi=\mp \frac{c \cdot \sin \left(\alpha_{L}-\beta_{R}\right)}{2}
$$

Detection probabilities depend on light intensities and thus on the square of probability amplitudes. Accordingly, (9), (10) yield the probabilities of joint detection events

$$
\begin{aligned}
& p\left(V_{\alpha_{L}} \wedge V_{\beta_{R}}\right)=p\left(H_{\alpha_{L}} \wedge H_{\beta_{R}}\right)=\frac{c^{2} \cdot \cos ^{2}\left(\alpha_{L}-\beta_{R}\right)}{4} \\
& p\left(V_{\alpha_{L}} \wedge H_{\beta_{R}}\right)=p\left(H_{\alpha_{L}} \wedge V_{\beta_{R}}\right)=\frac{c^{2} \cdot \sin ^{2}\left(\alpha_{L}-\beta_{R}\right)}{4},
\end{aligned}
$$

where the independence of (9), (10) against any common rotation of both analyzers is exploited. Since in case of $\alpha_{L}=\beta_{R}$ half of the joint detection counts belong to $V_{\alpha_{L}} \wedge V_{\beta_{R}}$ events while the other half is devoted to $H_{\alpha_{L}} \wedge H_{\beta_{R}}$ occurrences, it becomes clear from (11) that $c^{2}=2$ is required to be compatible with finding 1). Obviously, the probability amplitudes (4) and (5) refer to single photons while the value of $c^{2}$ ensures that joint detection probabilities refer to pairs of polarization-entangled photons. Thus Equation (11) are equivalent to the familiar results [12] 


$$
\begin{aligned}
& p\left(V_{\alpha_{L}} \wedge V_{\beta_{R}}\right)=p\left(H_{\alpha_{L}} \wedge H_{\beta_{R}}\right)=\cos ^{2}\left(\alpha_{L}-\beta_{R}\right) / 2 \\
& p\left(V_{\alpha_{L}} \wedge H_{\beta_{R}}\right)=p\left(H_{\alpha_{L}} \wedge V_{\beta_{R}}\right)=\sin ^{2}\left(\alpha_{L}-\beta_{R}\right) / 2 .
\end{aligned}
$$

Accordingly, for type-II SPDC

$$
\begin{aligned}
& p\left(V_{\alpha_{L}} \wedge V_{\beta_{R}}\right)=p\left(H_{\alpha_{L}} \wedge H_{\beta_{R}}\right)=\sin ^{2}\left(\alpha_{L}-\beta_{R}\right) / 2 \\
& p\left(V_{\alpha_{L}} \wedge H_{\beta_{R}}\right)=p\left(H_{\alpha_{L}} \wedge V_{\beta_{R}}\right)=\cos ^{2}\left(\alpha_{L}-\beta_{R}\right) / 2
\end{aligned}
$$

results. Each of the equation sets (12) or (13) comprise the probabilities of four joint detection outcomes for the respective type of polarization entanglement. They comply with the predictions of the non-local approach.

Excluding missing detections, the occurrence of $V_{\alpha_{L}}$ implies either a concurrent $H_{\beta_{R}}$ or $V_{\beta_{R}}$ detection. Thus from (12) the relations

$$
p\left(V_{\alpha_{L}}\right)=p\left(V_{\alpha_{L}} \wedge\left(V_{\beta_{R}} \vee H_{\beta_{R}}\right)\right)=p\left(V_{\alpha_{L}} \wedge V_{\beta_{R}}\right)+p\left(V_{\alpha_{L}} \wedge H_{\beta_{R}}\right)
$$

hold in accordance with the experimental observations. Thus

$$
p\left(V_{\alpha_{L}}\right)=p\left(H_{\alpha_{L}}\right)=p\left(V_{\beta_{R}}\right)=p\left(H_{\beta_{R}}\right)=0.5
$$

is valid for arbitrary analyzer alignments $\alpha_{L}, \beta_{R}$, as stated above. For the comparison with experimental results, conditional probabilities are of interest, i.e. the probabilities of a $V_{\beta_{R}}$ or $H_{\beta_{R}}$ event given either a $V_{\alpha_{L}}$ or a $H_{\alpha_{L}}$ detection event is observed. For instance for type-I SPDC

$$
p\left(V_{\beta_{R}} \mid V_{\alpha_{L}}\right)=\frac{p\left(V_{\alpha_{L}} \wedge V_{\beta_{R}}\right)}{p\left(V_{\alpha_{L}}\right)}=\cos ^{2}\left(\alpha_{L}-\beta_{R}\right)
$$

results. Therefore, (12) correspond with the conditional probabilities

$$
\begin{aligned}
& p\left(V_{\beta_{R}} \mid V_{\alpha_{L}}\right)=p\left(H_{\beta_{R}} \mid H_{\alpha_{L}}\right)=\cos ^{2}\left(\alpha_{L}-\beta_{R}\right) \\
& p\left(H_{\beta_{R}} \mid V_{\alpha_{L}}\right)=p\left(V_{\beta_{R}} \mid H_{\alpha_{L}}\right)=\sin ^{2}\left(\alpha_{L}-\beta_{R}\right)
\end{aligned}
$$

In case of type-II SPDC, the squared sine and cosine functions on the right of (17) have to be interchanged.

Note that because of symmetry of the local entanglement model, the allocation of the $\alpha$ and $\beta$ analyzer settings in (12) and (13) is arbitrary. Obviously, the first equations of (17) are equivalent to

$$
\begin{aligned}
& p\left(V_{\beta_{L}} \mid V_{\alpha_{R}}\right)=\cos ^{2}\left(\alpha_{R}-\beta_{L}\right) \\
& p\left(V_{\beta_{L}} \mid H_{\alpha_{R}}\right)=\sin ^{2}\left(\alpha_{R}-\beta_{L}\right)
\end{aligned}
$$

Experiments can only yield estimates of joint probabilities. In case of enough detection events, specific relations between joint detection counts map respective relations between probabilities. Accordingly, (14), (16) enable an immediate estimate of the conditional probability $p\left(V_{\beta_{R}} \mid V_{\alpha_{L}}\right)$ :

$$
p\left(V_{\beta_{R}} \mid V_{\alpha_{L}}\right) \approx \frac{n\left(V_{\alpha_{L}} \wedge V_{\beta_{R}}\right)}{n\left(V_{\alpha_{L}} \wedge V_{\beta_{R}}\right)+n\left(V_{\alpha_{L}} \wedge H_{\beta_{R}}\right)}
$$


where $n\left(V_{\alpha_{L}} \wedge V_{\beta_{R}}\right)$ denotes the number of recorded $\left(V_{\alpha_{L}} \wedge V_{\beta_{R}}\right)$ joint detections, for example. Equation (19) depends not on the $V_{\alpha_{L}}$ efficiency, whereas the estimate

$$
p\left(V_{\alpha_{L}} \mid V_{\beta_{R}}\right) \approx \frac{n\left(V_{\alpha_{L}} \wedge V_{\beta_{R}}\right)}{n\left(H_{\alpha_{L}} \wedge V_{\beta_{R}}\right)+n\left(V_{\alpha_{L}} \wedge V_{\beta_{R}}\right)}
$$

is insensitive to $V_{\beta_{R}}$. According to (14), the denominators of (19) and (20) equal $n\left(V_{\alpha_{L}}\right)$ and $n\left(V_{\beta_{R}}\right)$, respectively. In case of sufficient detection counts,

$$
p\left(V_{\beta_{R}} \mid V_{\alpha_{L}}\right) \approx p\left(V_{\alpha_{L}} \mid V_{\beta_{R}}\right)
$$

can be expected from (15), (19), and (20).

The probabilities of (11) to (18) were derived without any reference to nonlocal phenomena or hidden variables. Nevertheless, they exhibit the same dependence on $\left(\alpha_{L}-\beta_{R}\right)$ as previous non-local theories, enabling the same violation of Bell's inequality [2] [4] [5] [7] [12]. Thus, the derivation of (12), (13) confirms that violations of the Bell inequality constitute no evidence of non-local phenomena in quantum mechanics. However, the rejection of non-locality doesn't mean that the local realistic model of EPR becomes appropriate for the interpretation of the above experiment. If "local realistic" means perfect statistical independence of separate preparation results regarding the elements of an entangled state, EPR would certainly fail. On the contrary, there are no objections if local realistic stands for the conjunction of quantum theoretical features of the measurement objects with applicable local measurement conditions. The presented approach also contradicts the idea that space-time separated preparations of entangled particles can explore non-commuting properties of a single element of the entangled system. Separated measurements on discernible entangled particles do not correspond with successive measurements on the very same quantum entity. There is no sign of incompleteness of quantum mechanics at all.

Discrimination between indiscernibility and entanglement of quantum particles is crucial for the local approach. Indiscernibility is a vital prerequisite for the creation of polarization entangled photon pairs. Separate polarization preparations rest on distinguishable photons. Different propagation directions and particularly spatial separation make polarization entangled photons distinguishable while maintaining their exceptional polarization interrelationship. Hence, entangled photons remain indiscernible exclusively with respect to their polarization characteristics. Therefore, the extraordinary correlations of joint photon polarization preparations are coupled exclusively to polarization entanglement carried by distinguishable photons, a situation best suited for the treatment by local models.

With respect to Figure 1, a sole characteristic of this experiment is worth to mention. Actually, this setup employs fixed polarization analyzers while the two polarization modulators rotate the polarization of the constituents of $\Psi_{I}$, or $\Psi_{I I}$ independently. The related results are consistent with preparations of un- 
distorted photons by rotating polarization analyzers. Therefore, such space-time separated manipulations of both entangled photons do not affect the pairwise polarization entanglement.

\section{Conclusion}

Exploring polarization-entangled photon systems exclusively within the polarization domain of the Hilbert space encourage doubtful statements regarding their deployment in space and time. The local explanation presented departs from this restriction, acknowledging the purely local nature of polarization preparations by remote polarizers. The treatment assumes strict polarization correlation between the constituents of each pair of entangled photons whereas the sequence of photon pairs features arbitrary polarization. The current model proposes statistical independence of probability amplitudes in case of space-time separated polarization preparations. Ordinary quantum mechanical treatment can then explain the extraordinary correlations between space-separated polarization preparations when imposed on polarization-entangled photons. Without involvement of hidden variables, the astonishing simple local treatment yields the same results with respect to joint detection probabilities as previous non-local attempts. At least for the type of Bell experiments discussed, violations of the Bell inequality by no means imply non-local quantum mechanic phenomena or the incompleteness of quantum mechanics.

\section{Acknowledgments}

The author likes to thank Fritz Paschke for his profound support in physical modeling.

\section{Conflicts of Interest}

The author declares no conflicts of interest regarding the publication of this paper.

\section{References}

[1] Einstein, A., Podolsky, Yu. and Rosen, N. (1935) Can Quantum-Mechanical Description of Physical Reality Be Considered Complete? Physical Review, 47, 777. https://doi.org/10.1103/PhysRev.47.777

[2] Bell, J.S. (1964) On the Einstein Podolsky Rosen Paradox. Physics Physique, 1, 195 290. https://doi.org/10.1103/PhysicsPhysiqueFizika.1.195

[3] Leggett, A.J. (2003) Nonlocal Hidden-Variable Theories and Quantum Mechanics: An Incompatibility Theorem. Foundations of Physics, 33, 1469-1493. https://doi.org/10.1023/A:1026096313729

[4] Weihs, G., Jennewein, T., Simon, C., Weinfurter, H. and Zeilinger, A. (1998) Violation of Bell's Inequality under Strict Einstein Locality Conditions. Physical Review Letters, 81, 5039. https://doi.org/10.1103/PhysRevLett.81.5039

[5] Berkovitz, J. (2016) Action at a Distance in Quantum Mechanics. In: Zalta, E.N., Ed., The Stanford Encyclopedia of Philosophy, Spring 2016 Edition, Metaphysics Research Lab, Stanford University, Stanford, 1-107. https://plato.stanford.edu/archives/spr2016/entries/qm-action-distance 
[6] Fine, A. (2020) The Einstein-Podolsky-Rosen Argument in Quantum Theory. In: Zalta, E.N., Ed., The Stanford Encyclopedia of Philosophy, Summer 2020 Edition, Metaphysics Research Lab, Stanford University, Stanford, 1-41. https://plato.stanford.edu/archives/sum2020/entries/qt-epr

[7] Myrvold, W., Genovese, M. and Shimony, A. (2021) Bell's Theorem. In: Zalta, E.N., Ed., The Stanford Encyclopedia of Philosophy, Fall 2021 Edition, Metaphysics Research Lab, Stanford University, Stanford, 1-80. https:/plato.stanford.edu/archives/fall2021/entries/bell-theorem

[8] Schrödinger, E. (1936) Probability Relations between Separated Systems. Mathematical Proceedings of the Cambridge Philosophical Society, 32, 446-452. https://doi.org/10.1017/S0305004100019137

[9] Griffiths, R.B. (2020) Nonlocality Claims Are Inconsistent with Hilbert-Space Quantum Mechanics. Physical Review A, 101, Article ID: 022117. https://doi.org/10.1103/PhysRevA.101.022117

[10] Jung, K. (2020) Polarization Correlation of Entangled Photons Derived without Using Non-Local Interactions. Frontiers in Physics, 8, Article No. 170. https://doi.org/10.3389/fphy.2020.00170

[11] Hess, K. (2018) Bell's Theorem and Instantaneous Influences at a Distance. Journal of Modern Physics, 9, 1573-1590. https://doi.org/10.4236/jmp.2018.98099

[12] Weihs, G. (1999) Ein Experiment zum Test der Bell'schen Ungleichung unter Einstein'scher Lokalität, Ph.D. Thesis, Universität Wien, Wien.

https://www.univie.ac.at/qfp/publications/thesis

[13] French, S. (2019) Identity and Individuality in Quantum Theory, The Stanford Encyclopedia of Philosophy, Zalta, E. N. ed., The Stanford Encyclopedia of Philosophy, Winter 2019 Edition, Metaphysics Research Lab, Stanford University, 1-39. https://plato.stanford.edu/archives/win2019/entries/qt-idind,

[14] Feynman, R., Leighton, R. and Sands, M. (2010) The Feynman Lectures on Physics Vol. III, Millennium Edition. Basic Books, New York.

[15] Tikochinsky, Y. (1988) Feynman Rules for Probability Amplitudes. International Journal of Theoretical Physics, 27, 543-549. https://doi.org/10.1007/BF00668836

[16] Bell, J.S. and Aspect, A. (2004) Speakable and Unspeakable in Quantum Mechanics: Collected Papers on Quantum Philosophy. Cambridge University Press, Cambridge. https://doi.org/10.1017/CBO9780511815676 